\title{
EDITORIAL
}

\section{Microbial reduction in the NICU: seeing the light}

Journal of Perinatology (2011) 31, 573-574; doi:10.1038/jp.2011.36

Healthcare-associated infections in the neonatal intensive care unit (NICU) are mostly a consequence of host-environmental interactions. The relative immuno-deficient state of the premature infant, in combination with the use of invasive procedures, sets the stage up for an amalgamation of factors leading to nosocomial sepsis, with potentially disastrous outcomes. Caregivers of the susceptible neonate are well aware of the reasons behind catheterrelated infections and have developed strategies to decrease them. ${ }^{1,2}$ Another important contributor to sepsis-related morbidity in the NICU is associated with the use of invasive mechanical ventilation, that is, ventilator-associated pneumonia (VAP). ${ }^{3}$

VAP in the NICU has been reported to have a variable incidence from a low of 2.7 to a high of 70 per 1000 ventilator days, worldwide. ${ }^{4-8}$ Part of the problem has been the lack of consensus for validation of the diagnosis of VAP in neonates. ${ }^{3,9}$ Investigators have used techniques such as non-bronchoscopic bronchoalveolar lavage $^{10}$ and Gram staining of tracheal aspirates ${ }^{11}$ to enable early diagnosis and therapy. The source of VAP is mostly secondary to endogenous oropharyngeal colonization and exogenous sources such as ventilator circuits, humidifiers and endotracheal tubes. ${ }^{3}$

In the article by Ryan $e t a l,{ }^{12}$ an innovative approach was taken to impact on the exogenous sources of microorganisms that could potentially colonize the airway and contribute to VAP. The investigators hypothesized that enhanced-ultraviolet germicidal irradiation (eUVGI; also called the Pathogen Control System, Vigilair Systems, North Tonawanda, NY, USA) placed in the NICU heating ventilation and air conditioning system would decrease heating ventilation and air conditioning and NICU environmental and neonatal tracheal microbial loads and VAP. The diagnosis of VAP for this study used the CDC criteria for age-specific definition of nosocomial pneumonia. The microorganisms detected were the smorgasbord of usual suspects in a NICU environment-Grampositive and Gram-negative bacteria, as well as fungi.

In this prospective, single-center study, eUVGI exposure resulted in a dramatic decline in the microbial load in the heating ventilation and air conditioning and NICU surfaces, within 3 days, and was sustained up to 10 months. Tracheal aspirate colonization, VAP and antibiotic use did decrease significantly too, but seemed to mostly level off after the first month of exposure to eUVGI. ${ }^{12}$

Among the limitations of the study are the facts that the intervention was not randomized or blinded to the caregivers. Although the investigators mention that several practices remained unchanged, other clinical practices such as earlier extubation did increase during the study period, ${ }^{12}$ which could impact on the results. In addition, data on presence of concomitant nosocomial sepsis and/or necrotizing enterocolitis during the study, which would influence antibiotic use in the NICU, were not available. Interestingly, in this study, the length of time of intubation did not correlate with the degree of colonization, as measured by the 'tracheal microbial-load index', ${ }^{12}$ but data were not available on how long the same endotracheal tube was kept in situ.

The data reported by Ryan $e t a l^{12}$ is intriguing enough to warrant a multi-center randomized controlled trial with prospective collection of data on additional outcomes for a more definitive answer. ${ }^{13}$ In addition, it would be important to evaluate the cost-effectiveness of such an approach to decrease nosocomial sepsis and attendant healthcare expenses. Till such time, elevation of head/lateral body positioning of ventilated infants, 3,14 using a non-invasive approach ('lose the tube') to neonatal ventilation, ${ }^{3,15,16}$ and the tried and true techniques of line, tube and hand hygiene ${ }^{1}$ should 'light' our path in the NICU.

\section{Conflict of interest}

The author declares no conflict of interest.

V Bhandari

Division of Perinatal Medicine, Department of Pediatrics, Yale University School of Medicine, New Haven, CT, USA E-mail: vineet.bhandari@yale.edu

\section{References}

1 Graham III PL. Simple strategies to reduce healthcare associated infections in the neonatal intensive care unit: line, tube, and hand hygiene. Clin Perinatol 2010; 37: 645-653.

2 Sandora TJ. Prevention of healthcare-associated infections in children: new strategies and success stories. Curr Opin Infect Dis 2010; 23: 300-305.

3 Garland JS. Strategies to prevent ventilator-associated pneumonia in neonates. Clin Perinatol 2010; 37: 629-643.

4 Apisarnthanarak A, Holzmann-Pazgal G, Hamvas A, Olsen MA, Fraser VJ. Ventilatorassociated pneumonia in extremely preterm neonates in a neonatal intensive care unit: characteristics, risk factors, and outcomes. Pediatrics 2003; 112: 1283-1289.

5 Gunlemez A, Atasay B, Guriz H, Aysev D, Arsan S. Multi-resistant viridans streptococcal pneumonia and sepsis in the ventilated newborn. Ann Trop Paediatr 2004; 24: 253-258. 
6 Pessoa-Silva CL, Richtmann R, Calil R, Santos RM, Costa ML, Frota AC et al. Healthcare-associated infections among neonates in Brazil. Infect Control Hosp Epidemiol 2004; 25: 772-777.

7 Petdachai W. Ventilator-associated pneumonia in a newborn intensive care unit. Southeast Asian J Trop Med Public Health 2004; 35: 724-729.

8 Geffers C, Baerwolff S, Schwab F, Gastmeier P. Incidence of healthcare-associated infections in high-risk neonates: results from the German surveillance system for very-low-birthweight infants. J Hosp Infect 2008; 68: 214-221.

9 Baltimore RS. The difficulty of diagnosing ventilator-associated pneumonia. Pediatrics 2003; 112: 1420-1421.

10 Koksal N, Hacimustafaoglul M, Celebi S, Ozakin C. Nonbronchoscopic bronchoalveolar lavage for diagnosing ventilator-associated pneumonia in newborns. Turk J Pediatr 2006; 48: 213-220

11 Katayama Y, Minami H, Enomoto M, Takano T, Hayashi S, Lee YK. Usefulness of Gram staining of tracheal aspirates in initial therapy for ventilator-associated pneumonia in extremely preterm neonates. J Perinatol 2010; 30: 270-274.
12 Ryan RM, Wilding GE, Wynn RJ, Welliver RC, Holm BA, Leach CL. Effect of enhanced ultraviolet germicidal irradiation in the heating ventilation and air conditioning system on ventilator-associated pneumonia in a neonatal intensive unit. J Perinatol 2011; 30: 607-614.

13 Bradley JS. Considerations unique to pediatrics for clinical trial design in hospital-acquired pneumonia and ventilator-associated pneumonia. Clin Infect Dis 2010; 51:(Suppl 1): S136-S143.

14 Aly H, Badawy M, El-Kholy A, Nabil R, Mohamed A. Randomized, controlled trial on tracheal colonization of ventilated infants: can gravity prevent ventilator-associated pneumonia? Pediatrics 2008; 122: 770-774.

15 Hentschel J, Brungger B, Studi K, Muhlemann K. Prospective surveillance of nosocomial infections in a Swiss NICU: low risk of pneumonia on nasal continuous positive airway pressure? Infection 2005; 33: 350-355.

16 Bhandari V. Nasal intermittent positive pressure ventilation in the newborn: review of literature and evidence-based guidelines. J Perinatol 2010; 30 $505-512$. 